Research Article

\title{
Electromagnetic Scattering of Electrically Large Ship above Sea Surface with SBR-SDFM Method
}

\author{
Lixin Guo and Tiantian Feng \\ School of Physics and Optoelectronic Engineering, Xidian University, Xian 710071, China \\ Correspondence should be addressed to Tiantian Feng; titife037@163.com
}

Received 7 July 2017; Accepted 25 September 2017; Published 26 October 2017

Academic Editor: Matteo Pastorino

Copyright (C) 2017 Lixin Guo and Tiantian Feng. This is an open access article distributed under the Creative Commons Attribution License, which permits unrestricted use, distribution, and reproduction in any medium, provided the original work is properly cited.

Hybrid scheme combining shooting and bouncing ray with semi-deterministic facet model is proposed to analyze composite scattering from ship-ocean scene in this study. This model can deal with complex electromagnetic interaction between ship and sea surface. Thus, scattering properties of composite ship-ocean scenes with influence of various parameters (such as incident angle and wind speed) can be studied and analyzed efficiently. Studying such properties is of significance for target detection and high-resolution radar imaging in sea environments. Accuracy and performance of this method are validated and evaluated by comparing with multilevel fast multipole method of FEKO for electrically small objects. All simulation results indicate that the proposed method is suitable for providing preliminary radar cross section prediction of electrically large composite model.

\section{Introduction}

Electromagnetic scattering of electrically large targets above rough sea surface is common military scene in target detection and tracking [1-3]. In recent years, extensive endeavors have been devoted to simulation of such complex composite scattering. Method of Moment (MoM) [4] is widely used numerical algorithm, which is adequately developed into fast algorithms, such as Generalized Forward-Backward Method (GFBM) $[5,6]$ and multilevel fast multipole algorithm (MLFMA) [7, 8]. However, such methods require considerable computational time when dealing with electrically large targets. Asymptotic methods such as Iterative Physical Optics (IPO) which contains a large number of iterations to ensure the accuracy also face poor computing efficiency. Traditional four-path model [9] uses complex reflection coefficient to evaluate coupling interaction of ship-ocean. However, actual sea surface is usually assumed to be two-scale rough surface model [10]: capillary waves modulated by gravity wave in large scale. Thus, scattering field is not only concentrated on specular direction. Besides, four-path model cannot consider mutual shade between ship and ocean. Shooting and Bouncing Ray (SBR) [11, 12] seems more flexible and suitable for such issue. With ray tracing capacity of SBR method, multiple interactions between ship and ocean can be determined. Nevertheless, SBR method requires more refined meshes involving capillary waves on the sea surface. This requirement enormously increases computing burden. For large-scope sea surfaces, semi-deterministic facet scattering model (SDFM) $[13,14]$ was developed in relevant publications. In this model, elementary radar echoes from respective facets are computed by a semideterministic scheme considering both specular and diffuse configurations.

This paper aims to establish feasible scheme that conforms to sea surface two-scale model by combining SDFM and SBR method. With SDFM considering Bragg scattering from capillary waves, sea surface can be meshed into larger triangle patches, which adapt well to SBR method for ray tracing between the ship and ocean. Additionally, based on improved backward SBR tracing process and kdtree acceleration treatment mentioned in [15], computation time rapidly decreases. In the proposed composite scattering model, SBR method is used to calculate scattering from multiple interactions of ship-ocean and scattering from ship, whereas scattering from sea surface is calculated by SDFM. Hybrid SBR-SDFM method should be time-saving and highly efficient to handle radar cross section calculation of electrically large ship-ocean composite model. 


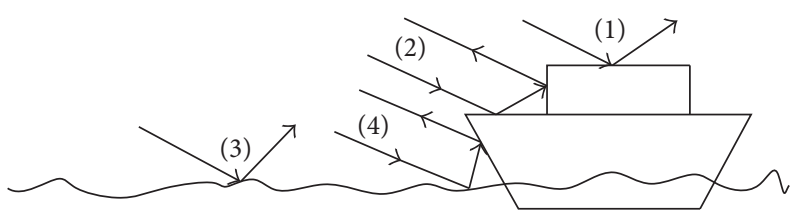

FIGURE 1: Illustration of composite scattering model.

\section{SBR-SDFM Method for Composite Scattering}

2.1. Composite Scattering Model of Ship-Ocean. Figure 1 shows simplified composite scattering model of ship above rough sea surface. Under framework of the SBR-SDFM method, composite scattering model is divided into four major parts.

(1) Single Scattering from Ship. This part is calculated equivalently by physical optics. During the computation, not only self-shade of ship but also shade to ship by sea surface should be taken into consideration. As shown in Figure 1, scattering calculation does not involve part of ship submerged in sea surface.

(2) Multiple Scattering from Ship. This part is calculated by SBR. Ship model usually contains numerous dihedral structures; these structures can generate large number of multiple reflections, which develop into major components of backscattered field. Hence, multiple scattering of ship should be considered.

(3) Single Scattering from Sea Surface. SDFM method is adopted to calculate scattering contribution of sea surface. In the theory of SDFM, sea wave is envisaged as a superposition of gravity wave configuration approximated by planar facets and capillary wave configuration added on the planar facets at resonant wavenumber. This assumption better conforms to the sea surface two-scale model than PO or SBR. Then, with Bragg scattering from capillary waves being considered separately, sea surface can be meshed into larger triangle patches, which can adapt well to the SBR method for ray tracing between ship and ocean. Thus, computing time is comparatively reduced. Furthermore, visibility of facets on the sea surface should be checked to eliminate shade from ship and other facets on the sea surface.

(4) Coupling Scattering between Ship and Sea Surface. This part will be discussed in Section 2.2 in detail.

Then, scattered field of composite model $\vec{E}^{\text {total }}$ can be developed by creating superposition of obtained scattered fields:

$$
\vec{E}^{\text {total }}=\vec{E}^{\text {ship }, 1}+\vec{E}^{\text {ship }, 2,3, \ldots}+\vec{E}^{\text {sea }, 1}+\vec{E}^{\text {sea-ship }, 1,2,3,4}
$$

where $\vec{E}^{\text {ship, } 1}, \vec{E}^{\text {ship,2,3,... }}, \vec{E}^{\text {sea, } 1}$, and $\vec{E}^{\text {sea-ship, } 1,2,3,4}$ are scattering field of four parts mentioned above, respectively.

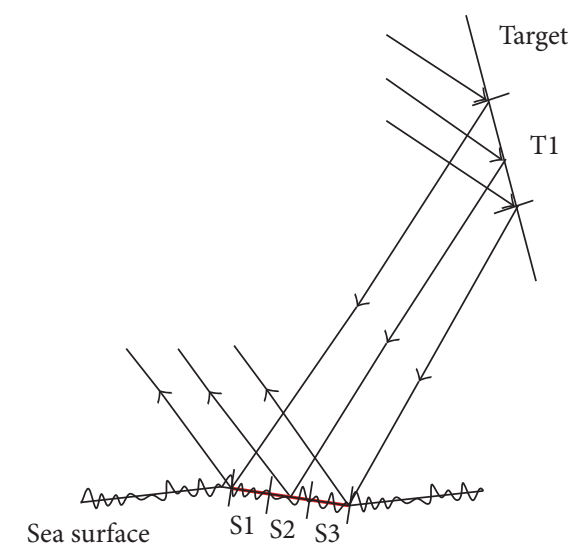

FIGURE 2: Illustration of ray tracing for coupling interaction of shipocean.

2.2. Coupling Scattering between Ship and Ocean. SBR method is applied to describe coupling of ship and sea surface (see Figure 2). There are four main ray paths included: shipsea, sea-ship, ship-sea-ship, and sea-ship-sea. First, the ship and the sea surface are meshed into triangle patches, which are numbered and stored into kd-trees, respectively. Taking ray path ship-sea as an example, given the incident wave direction, patches on the ship model illuminated directly by the incident wave will be identified (i.e., triangle T1) and stored into computer memory as an array. Then the ray starts from illuminated patches on the ship and propagates along the reflected direction, implementing intersection tests with previously built $\mathrm{kd}$-tree of sea surface to determine illuminated patches on the sea surface. Once one patch is found to be illuminated (i.e., triangle S2), backward ray tracing will be performed on its neighbors (i.e., triangles S1 and S3; their IDs are always adjacent to illuminated patches) to check their visibility. Finally, calculation will be performed on far field scattering of all illuminated patches on the sea surface.

In this study, scattering field from sea surface is computed based on SDFM. Bragg scattering field from each facet can be obtained by Fuks' model, with fair approximation that Bragg wave is a single frequency sinusoidal wave to simplify computation, and the polarization factor is modulated by gravity wave. The Bragg scattering field can be expressed as follows:

$$
\vec{E}_{p q}^{\text {facet }}\left(\widehat{k}_{i}, \widehat{k}_{s}\right)=2 \pi \frac{\exp (i k R)}{i R} S_{p q}\left(\widehat{k}_{i}, \widehat{k}_{s}\right)
$$

where $\widehat{k}_{i}$ and $\widehat{k}_{s}$ represent the combined vectors of incident and scattered wave number, respectively; $R$ is the distance from the observation to the facet centers; $k$ is the electromagnetic wave number; $p$ and $q$ are the polarization of the incident and scattered wave; $S_{p q}$ denotes the scattering amplitude that can be expressed as

$$
\begin{aligned}
S_{p q} & \left(\widehat{k}_{i}, \widehat{k}_{s}\right) \\
\quad & =\frac{k^{2}(1-\varepsilon)}{8 \pi^{2}} F_{p q} \iint \xi(\vec{r}) \exp (-i \vec{q} \cdot \vec{r}) d \vec{r},
\end{aligned}
$$




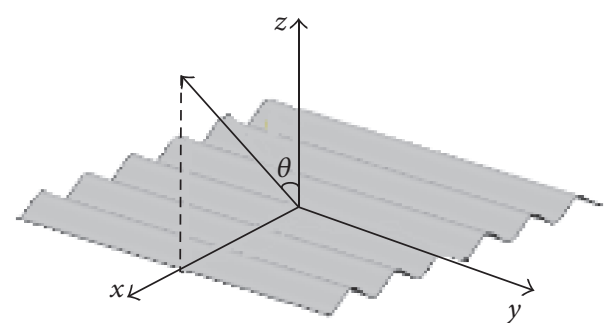

FIGURE 3: Illustration of a single frequency sinusoidal wave model.

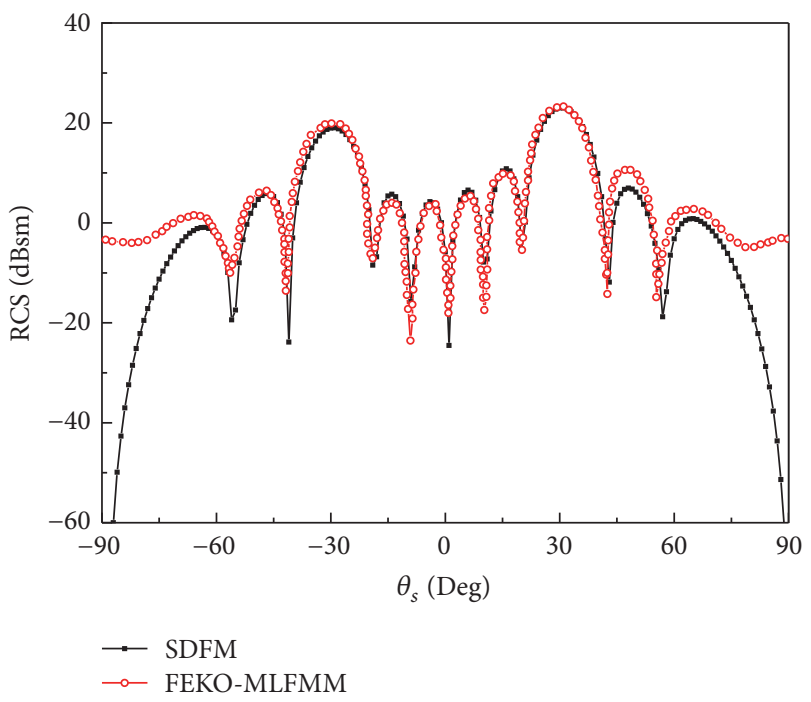

Figure 4: Bistatic RCS results for the facet (HH-polarization).

where $\varepsilon$ is the relative permittivity of the sea water. $\vec{q}$ is defined by $\vec{q}=k\left(\widehat{k}_{i}-\widehat{k}_{s}\right)$, and $k$ is the electromagnetic wave number. $F_{p q}$ is the polarization factor.

$\xi(\vec{r})$ in (3) represents the fluctuations of the capillary on the facet, which is envisaged to be a sinusoidal wave that can result in the Bragg resonance and it can be written as

$$
\xi(\vec{r}, t)=B\left(\vec{k}_{c}\right) \sin \left(\vec{k}_{c} \cdot \vec{\rho}_{c}-\omega_{c} t\right),
$$

where $\vec{k}_{c}, \omega_{c}$ are the wavenumber and circle frequency of the Bragg resonance and $\vec{\rho}_{c}$ is the coordinate of the respective point on the facet. $B\left(\vec{k}_{c}\right)=2 \pi \sqrt{S_{P M}\left(\vec{k}_{c}\right) / \Delta S}$ is the amplitude of the sinusoidal short wave, $S_{P M}\left(\vec{k}_{c}\right)$ is the sea spectrum, and $\Delta S$ is the area of each facet.

We have performed simulation for an individual facet $(6 \lambda \times 6 \lambda)$ on the sea surface (see Figure 3$)$ to verify the correctness of the model. Consider a sinusoidal wave expressed by $\xi=0.015 \cos \left(2 \pi x_{g} / \Lambda\right)$, where $x_{g}$ is the size of the facet and $\Lambda=\lambda /\left(2 \sin \theta_{i}\right)$ is the spatial wavelength satisfying the Bragg resonance. The dielectric constant of the sea surface is $(71.603,50.657)$ and the incident wave frequency is set to $2.0 \mathrm{GHz}$. The incident direction is set by $\theta_{i}=30^{\circ}$, $\varphi_{i}=0^{\circ}$. Figures 4 and 5 show numerical results of SDFM compared with those simulated by MLFMM in commercial software FEKO. Results of SDFM are consistent with those of

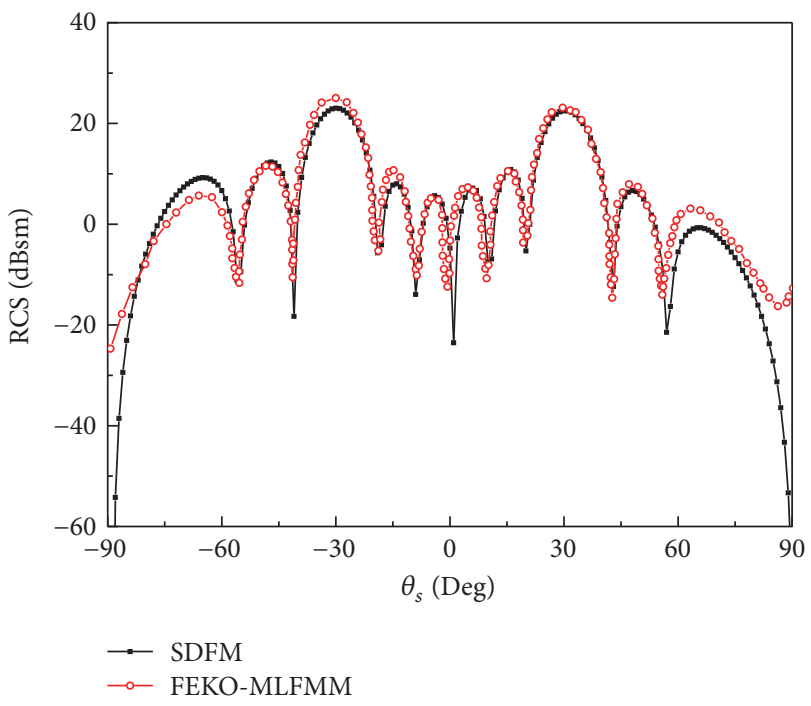

FIGURE 5: Bistatic RCS results for the facet (VV-polarization).

TABLE 1: Runtime performances of different methods.

\begin{tabular}{lcc}
\hline Method & SDFM & MLFMM (FEKO) \\
\hline Runtime (s) & 49.864 & 4210.692 \\
\hline
\end{tabular}

MLFMM. Table 1 presents the computational time of results in Figure 4. The computer configuration is $\operatorname{Intel}(\mathrm{R}) \operatorname{Xeon}(\mathrm{R})$ CPU (2.1 GHz, 32.0 GB).

As for the ray path sea-ship, instead of patches on the ship, patches on the sea surface illuminated directly by the incident wave will be determined first, and the ray tracing process will be implemented from sea surface to ship. Finally, scattering field of illuminated patches on the ship will be calculated by PO. Scattering field approximated by PO integral can be written as follows:

$$
\begin{aligned}
\vec{E}^{s}= & \frac{j k \exp (-j k R)}{4 \pi R} \\
& \cdot \iint \eta \cdot \widehat{k}_{s} \times\left(\widehat{k}_{s} \times \vec{J}\right) \exp j k \widehat{k}_{s} \cdot \vec{r}^{\prime} d s^{\prime},
\end{aligned}
$$

where $\eta=\left(\varepsilon_{0} / \mu_{0}\right)^{1 / 2} ; \vec{J}$ is the current density at $\vec{r}^{\prime}$ on the patch.

The high order coupling interaction of sea-ship-sea or ship-sea-ship is similar to the second-order interaction described above. The difference is that high order coupling interaction requires one more ray tracing process.

2.3. Multiple Scattering of Ship. There are some simulation results to verify the necessity of taking multiple scattering of the ship into account. Figure 6 displays dimensions of the ship. Figure 7 shows monostatic scattering results of the ship; results include both first-order scattering and second-order scattering.

Results present visible difference. When multiple scattering of the ship is considered, the backscattering field will be significantly enhanced. 


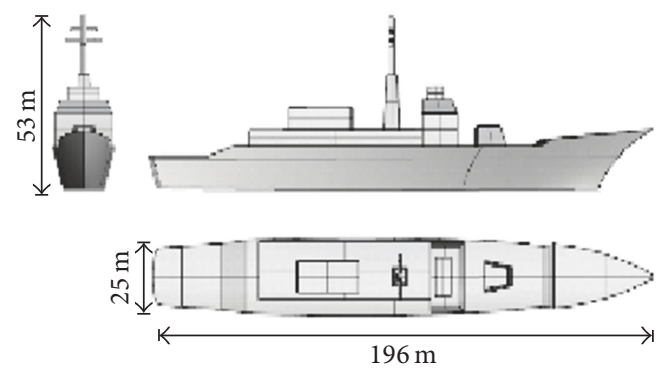

FIGURE 6: Illustration of dimensions of the ship.

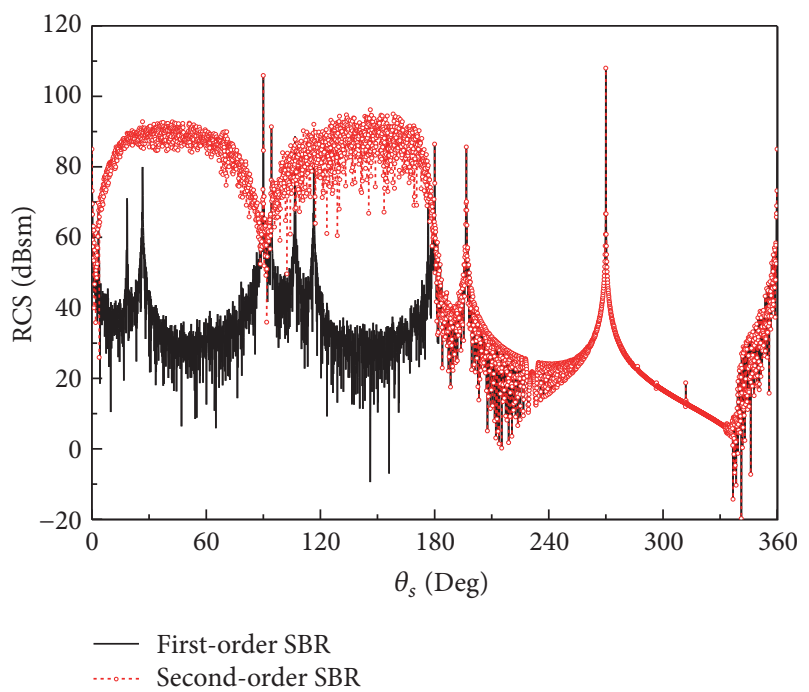

FIGURE 7: Monostatic RCS results for the ship with the incident plane at $10 \mathrm{GHz}$.

\section{Results and Discussion}

\subsection{Validation for the Composite Scattering Model}

3.1.1. Cube on Flat Plate. The composite model of a flat plate $\left(0.36 \times 0.36 \mathrm{~m}^{2}\right)$ with a cube located over at height of $0.03 \mathrm{~m}$ is simulated at frequency of $10 \mathrm{GHz}$ for $\mathrm{HH}$-polarization (see Figure 8). Figure 9 shows bistatic RCS of the model when side length of the cube is $0.03 \mathrm{~m}$, and the incident angles are set to $\theta_{i}=45^{\circ}, \varphi_{i}=0^{\circ}$. The monostatic RCS is shown in Figure 10. The results of SBR method agree well with results based on MLFMM. Furthermore, higher accuracy is obtained compared with the four-path model.

3.1.2. Sphere on Rough Sea Surface. The diameter of the sphere is $0.3 \mathrm{~m}$ and the sphere is above the sea surface by $0.15 \mathrm{~m}$ (see Figure 11). The sea area is $1.8 \times 1.8 \mathrm{~m}^{2}$, and the wind speed is $U=0.5 \mathrm{~m} / \mathrm{s}$. The incident angle is $\theta_{i}=30^{\circ}$, whereas the frequency of the incident wave is $4 \mathrm{GHz}$. Both the sphere and sea surface are assumed to be perfect electronic conductor. Figure 12 shows the bistatic RCS results of the model for $\mathrm{HH}$-polarization. The simulation results calculated by our method are in good agreement with those by FEKOMLFMM.

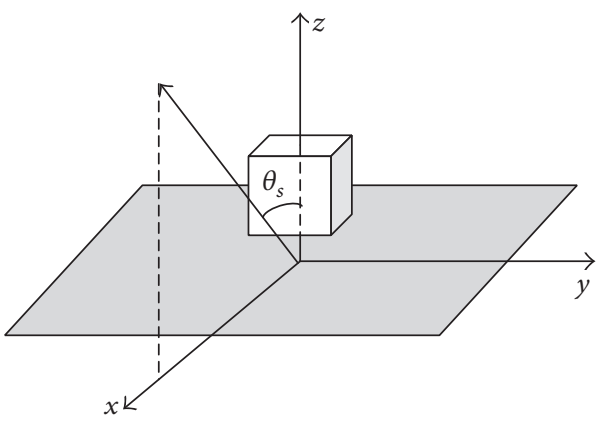

FIgURE 8: Illustration of a cube on flat plate.

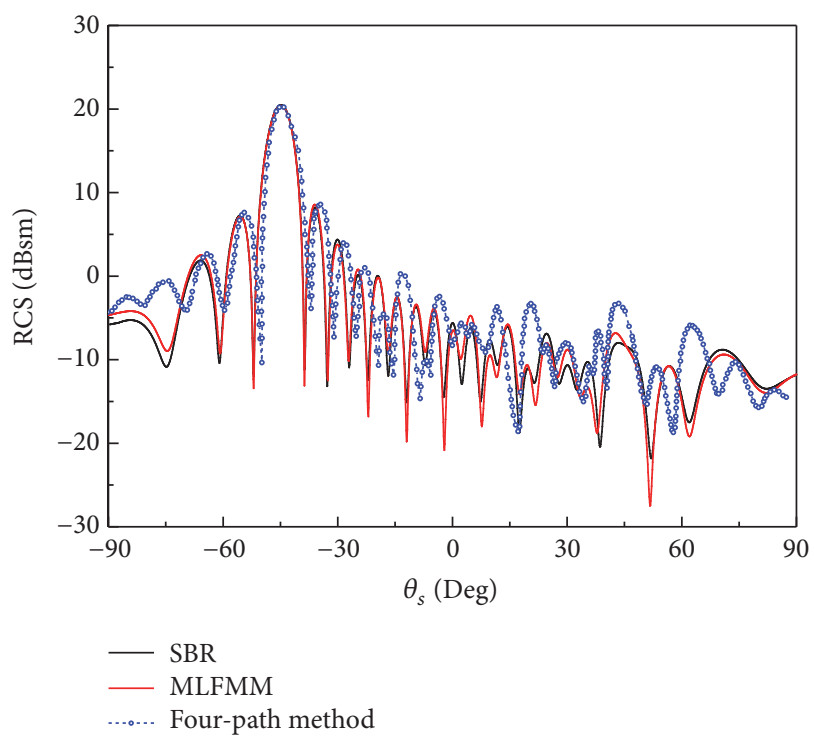

FIGURE 9: Bistatic RCS results for $0.03 \mathrm{~m}$ cube on flat plate.

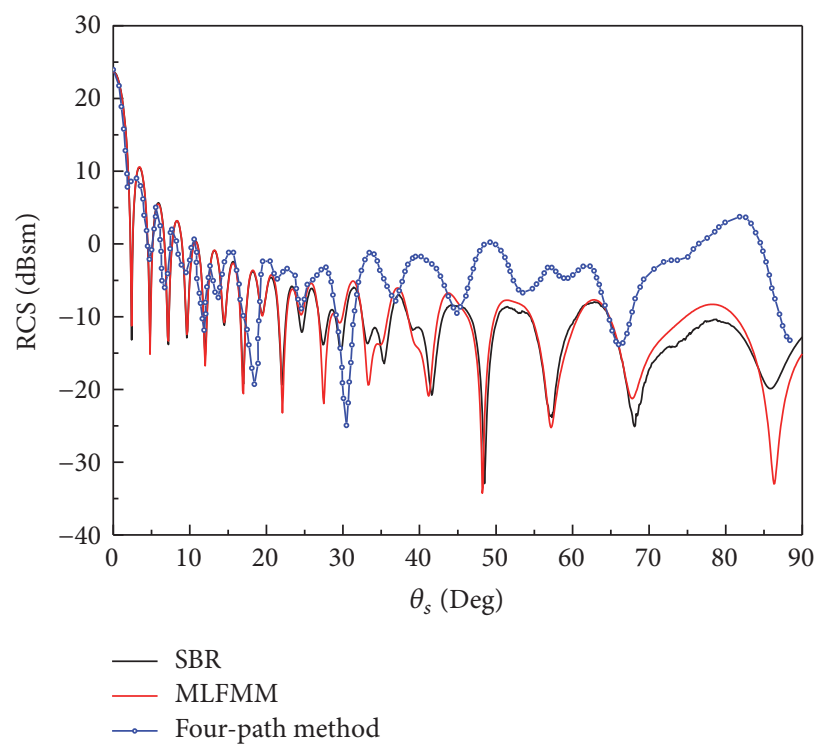

FIGURE 10: Monostatic RCS results for $0.03 \mathrm{~m}$ cube on flat plate. 


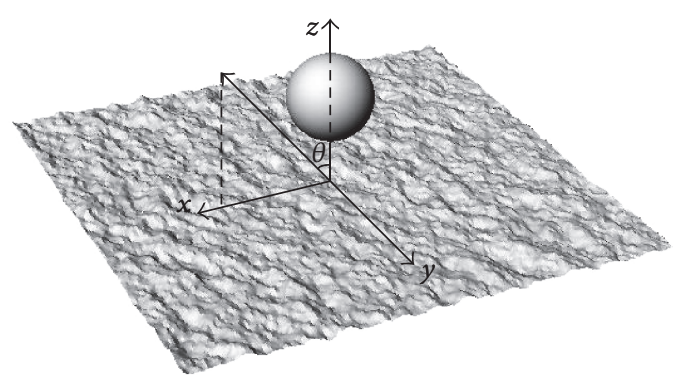

FIGURE 11: Illustration of a sphere on flat plate.

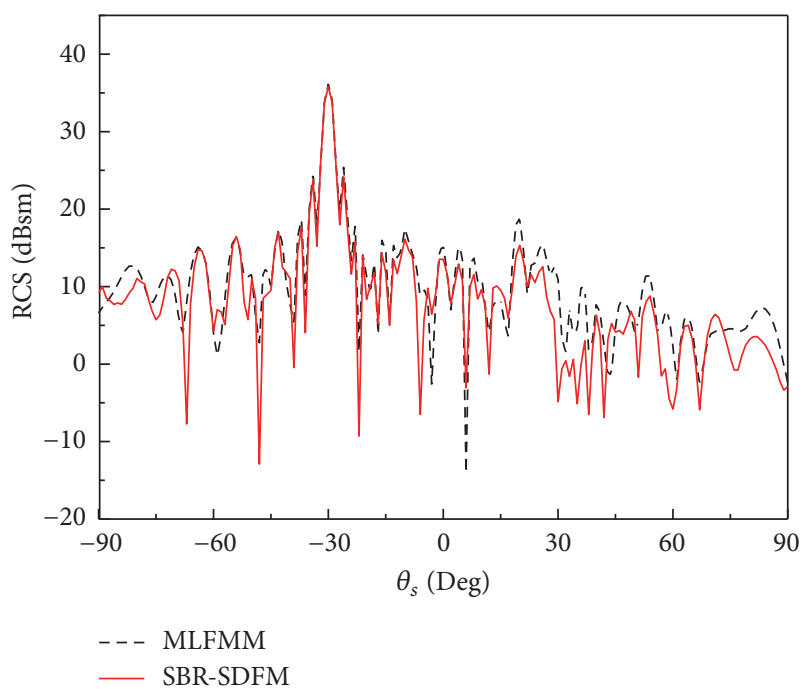

FIGURE 12: Bistatic RCS for a sphere above sea surface.

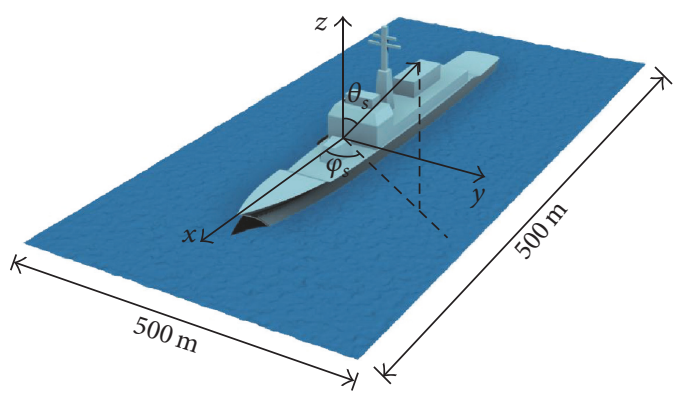

FIGURE 13: Illustration of the composite model of ship-ocean.

3.2. Composite Scattering of the Ship-Ocean. In the following, SBR-SDFM method is applied to the RCS prediction of the battle cruiser above the sea surface. As presented in Figure 13 (dimensions of the ship are the same as those in Figure 6), large size of model causes almost impossible calculation and analysis of scattering problem using numerical algorithm at high frequencies.

In our simulation, the incident wave frequency is fixed at $10 \mathrm{GHz}$. The permittivity of the sea surface is (65.4219, 34.9864). First, bistatic RCS for $\mathrm{HH}$-polarization is calculated with the incident direction set at $\theta_{i}=60^{\circ}, \varphi_{i}=90^{\circ}$, and the scattering direction is $\theta_{s}=-90^{\circ} \sim 90^{\circ}, \varphi_{s}=90^{\circ}$. The wind speed is selected by $U=5 \mathrm{~m} / \mathrm{s}$. Figure 14 illustrates

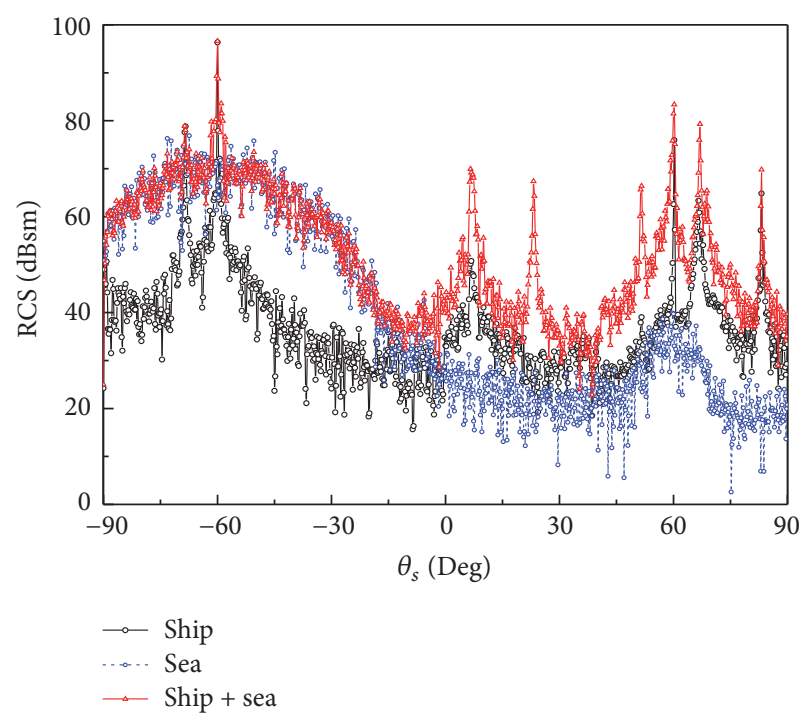

FIGURE 14: Bistatic RCS results of HH-polarization for the shipocean model.

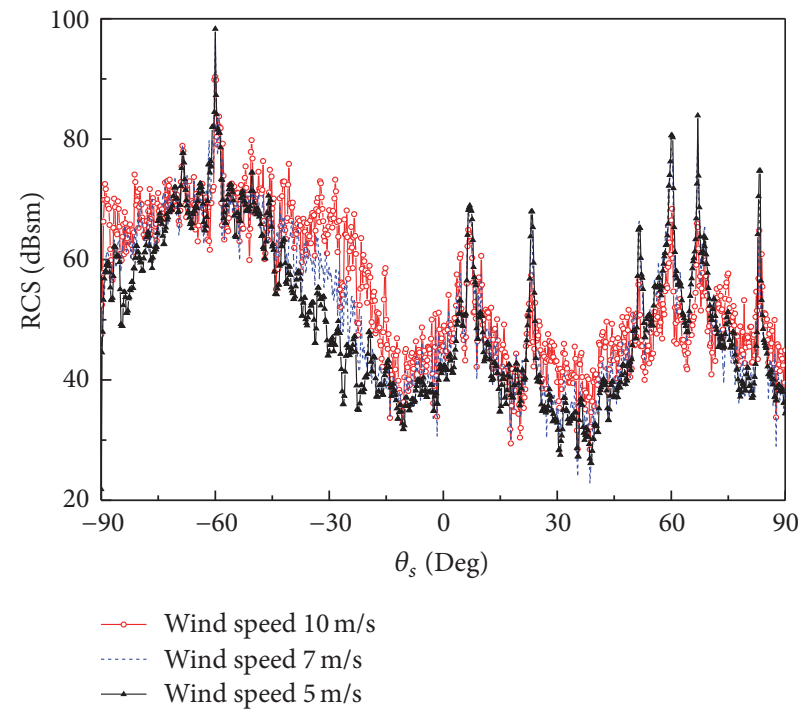

Figure 15: Bistatic RCS results for HH-polarization of the shipocean model at different wind speed.

bistatic RCS results. For this scenario, flank of the ship and the sea surface form strong coupling, which can significantly increase the backscattered field intensity. It can be seen from the results that when scattering angle varies from $\theta_{s}=0^{\circ}$ to $\theta_{s}=90^{\circ}$, scattering from both ship and coupling interaction preponderate in the total scattered field because of dihedral reflectors on the ship and dihedral reflectors formed by flank of the ship and the sea surface. Nevertheless, in the specular direction, scattering from the sea surface dominates the total scattering field.

As is known, the wind speed is a principal factor that influences the composite scattering field. The simulation results of bistatic RCS for the $\mathrm{HH}$-polarization in different wind speeds $5 \mathrm{~m} / \mathrm{s}, 7 \mathrm{~m} / \mathrm{s}$, and $10 \mathrm{~m} / \mathrm{s}$ are shown in Figure 15 . The incident wave slants at $\theta_{i}=60^{\circ}, \varphi_{i}=90^{\circ}$, and the 


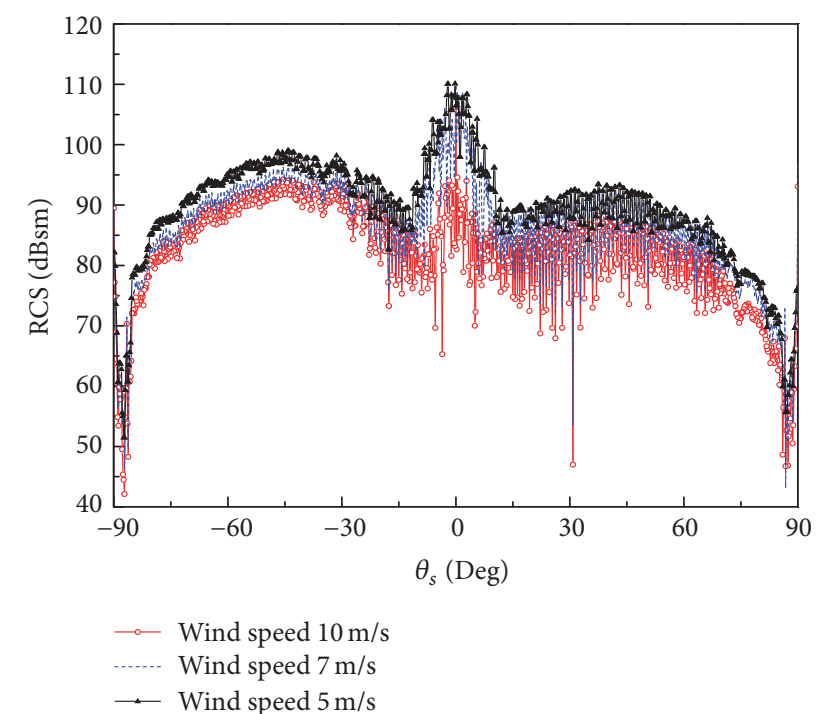

FIGURE 16: Monostatic RCS results for HH-polarization of the shipocean model at different wind speed.

scattering direction is $\theta_{s}=-90^{\circ} \sim 90^{\circ}, \varphi_{s}=90^{\circ}$. As the wind speed increases, the fluctuation of sea surface becomes stronger, which can attenuate the scattering intensity in specular direction but enhance that in diffuse direction. From the comparison results in Figure 15, one can see that scattering field intensity in most diffuse directions increases and the scattering field intensity in specular direction decreases, as the wind speed increases.

Figure 16 indicates the monostatic RCS results of the ship-ocean model at different wind speed. The incident and scattering angles are set by $\theta_{s}=\theta_{i}, \varphi_{s}=\varphi_{i}=$ $90^{\circ}$. As the wind speed decreases, specular scattering from sea surface is enhanced. This phenomenon consequently amplifies the coupling interaction between ship and ocean, further increasing the composite scattering.

\section{Conclusion}

This paper proposes suitable and efficient hybrid SBR-SDFM method for calculation of composite scattering of electrically large ship above sea surface. SBR and SDFM are, respectively, applied to the ship and sea surface. For the key problem coupling interaction between the ship and the sea surface, SBR is used to provide accurate simulation. Results for the validation of the method indicate good agreement with exact numerical method of FEKO.

\section{Conflicts of Interest}

The authors declare that they have no conflicts of interest.

\section{Acknowledgments}

This work was supported in part by the National Science Foundation for Distinguished Young Scholars of China (Grant no. 61225002), the Foundation for Innovative Research Groups of the National Natural Science Foundation of China (Grant no. 61621005), the National Natural Science Foundation of China (Grant no. 61431010), and the National Science Foundation of China (Grant no. 61501361).

\section{References}

[1] V. Fabbro, P. F. Combes, and N. Guillet, "Apparent radar cross section of a large target illuminated by a surface wave above the sea," Progress in Electromagnetics Research, vol. 50, pp. 41-60, 2005.

[2] W.-J. Ji and C.-M. Tong, "Bistatic scattering from twodimensional dielectric ocean rough surface with a pec object partially embedded by using the G-SMCG method," Progress in Electromagnetics Research, vol. 105, pp. 119-139, 2010.

[3] A. Baussard, M. Rochdi, and A. Khenchaf, "PO/MEC-based scattering model for complex objects on a sea surface," Progress in Electromagnetics Research, vol. 111, pp. 229-251, 2011.

[4] S. M. Rao, D. R. Wilton, and A. W. Glisson, "lectromagnetic scattering by surfaces of arbitrary shape," IEEE Transactions on Antennas and Propagation, vol. 30, no. 3, pp. 409-412, 1982.

[5] M. Pino, L. Landesa, J. L. Rodriguez, F. Obelleiro, and R. J. Burkholder, "The generalized forward-backward metod for analyzing the scattering from targets on ocean-like rough surfaces," IEEE Transactions on Antennas and Propagation, vol. 47, no. 6, pp. 961-969, 1999.

[6] Y. Jin and Z. Li, "Numerical simulation of radar surveillance for the ship target and oceanic clutters in two-dimensional model," Radio Science, vol. 38, no. 3, pp. 1045-1050, 2003.

[7] W. Yang, Z. Q. Zhao, C. H. Qi, W. Liu, and Z. P. Nie, "Iterative hybrid method for electromagnetic scattering from a 3-d object above a 2-d random dielectric rough surface," Progress in Electromagnetics Research, vol. 117, pp. 435-448, 2011.

[8] O. Ergul, "Parallel implementation of mlfma for homogeneous objects with various material properties," Progress in Electromagnetics Research, vol. 121, pp. 505-520, 2011.

[9] J. T. Johnson, "A study of the four-path model for scattering from an object above a half space," Microwave and Optical Technology Letters, vol. 30, no. 2, pp. 130-134, 2001.

[10] F. G. Bass and I. M. Fuks, Wave Scattering from Statistically Rough Surfaces, Pergamon Press, 1979.

[11] H. Ling, "Shooting and Bouncing Rays: Calculating the RCS of an Arbitrarily Shaped Cavity," IEEE Transactions on Antennas and Propagation, vol. 37, no. 2, pp. 194-205, 1989.

[12] L.-X. Guo and T.-Q. Fan, "Octree based backward SBR-PO method for electromagnetic scattering of electrically large target," in Proceedings of the 2015 1st IEEE International Conference on Computational Electromagnetics, ICCEM 2015, pp. 300-305, hkg, February 2015.

[13] Y. Wei, L. Guo, and J. Li, "Numerical simulation and analysis of the spiky sea clutter from the sea surface with breaking waves," Institute of Electrical and Electronics Engineers. Transactions on Antennas and Propagation, vol. 63, no. 11, pp. 4983-4994, 2015.

[14] F. G. Bass, I. M. Fuks, A. I. Kalmykov, I. E. Ostrovsky, and A. D. Rosenberg, "Very high frequency radiowave scattering by a disturbed sea surface part i: scattering from a slightly disturbed boundary," IEEE Transactions on Antennas and Propagation, vol. AP-16, no. 5, pp. 554-559, 1968.

[15] T.-Q. Fan, L.-X. Guo, B. Lv, and W. Liu, "An improved backward SBR-PO/PTD hybrid method for the backward scattering prediction of an electrically large target," IEEE Antennas and Wireless Propagation Letters, vol. 15, pp. 512-515, 2016. 


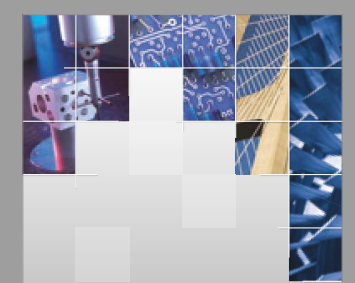

\section{Enfincering}
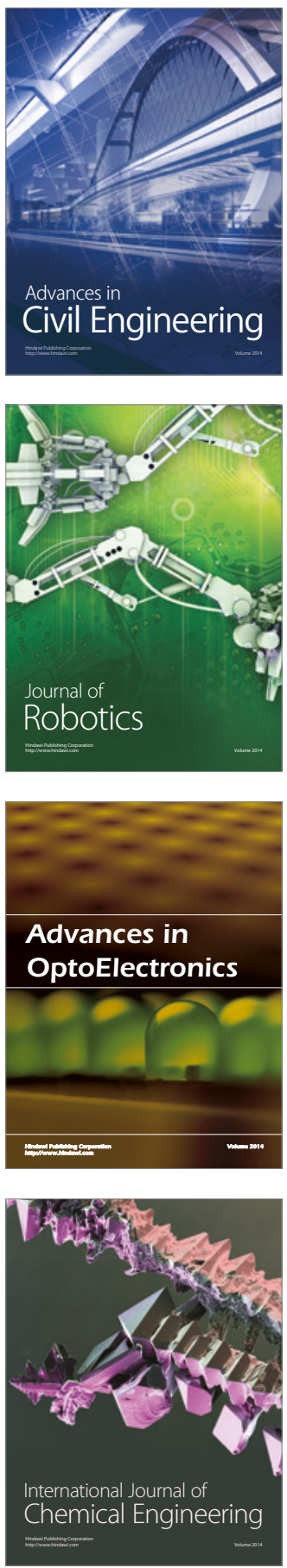

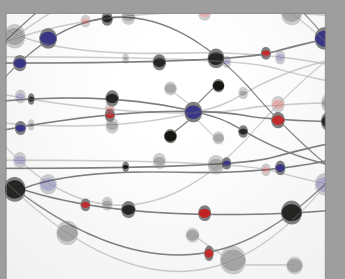

The Scientific World Journal

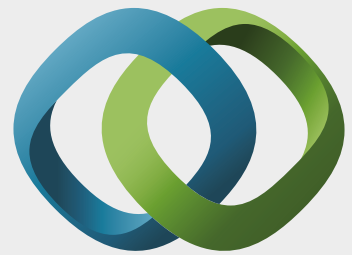

\section{Hindawi}

Submit your manuscripts at

https://www.hindawi.com
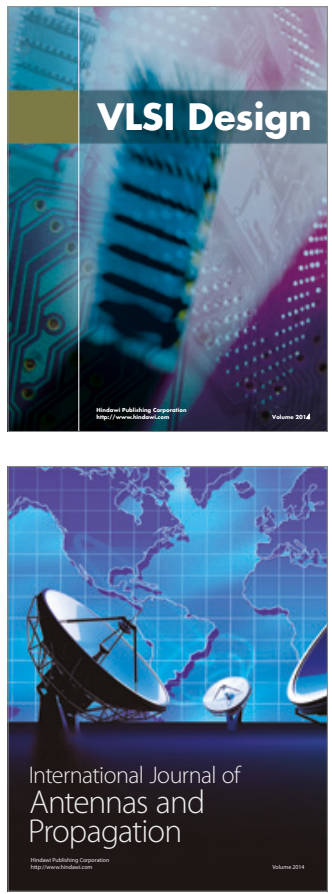

\section{Rotating}

Machinery
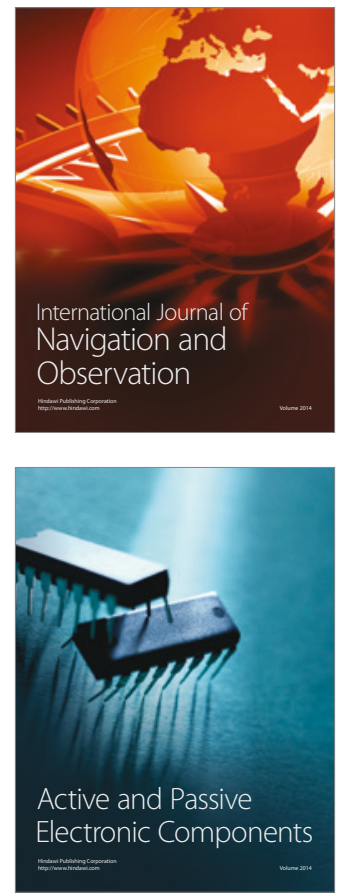
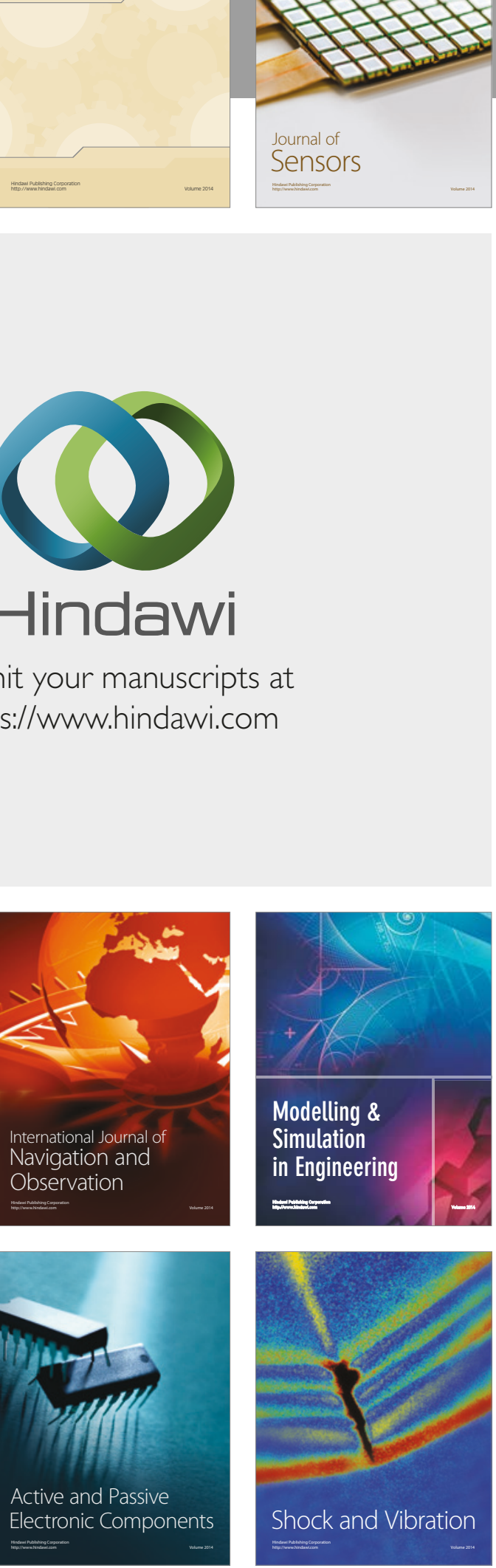
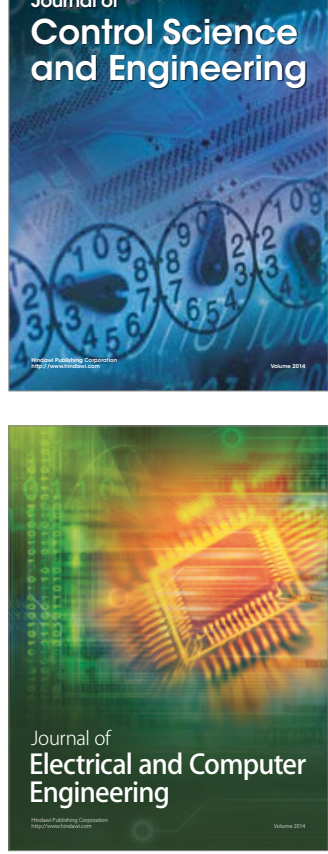

Distributed

Journal of

Control Science

and Engineering
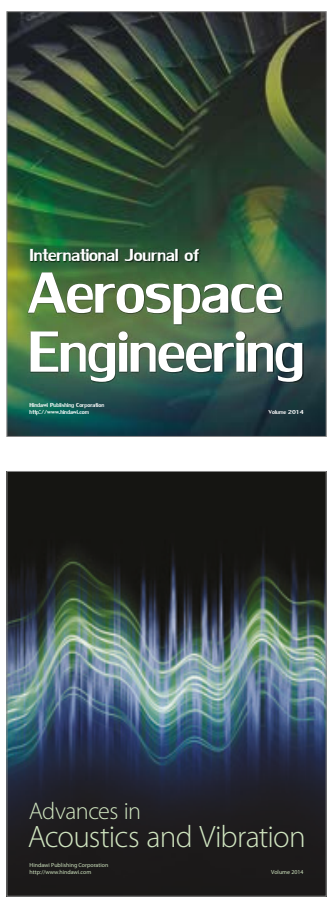

Sensor Networks 